\title{
Use of personalized cancer drugs runs ahead of the science
}

Clinical trial finds no benefit in speculative prescriptions of treatments tailored to the genetics of individual tumours.

\section{Asher Mullard}

17 September 2015

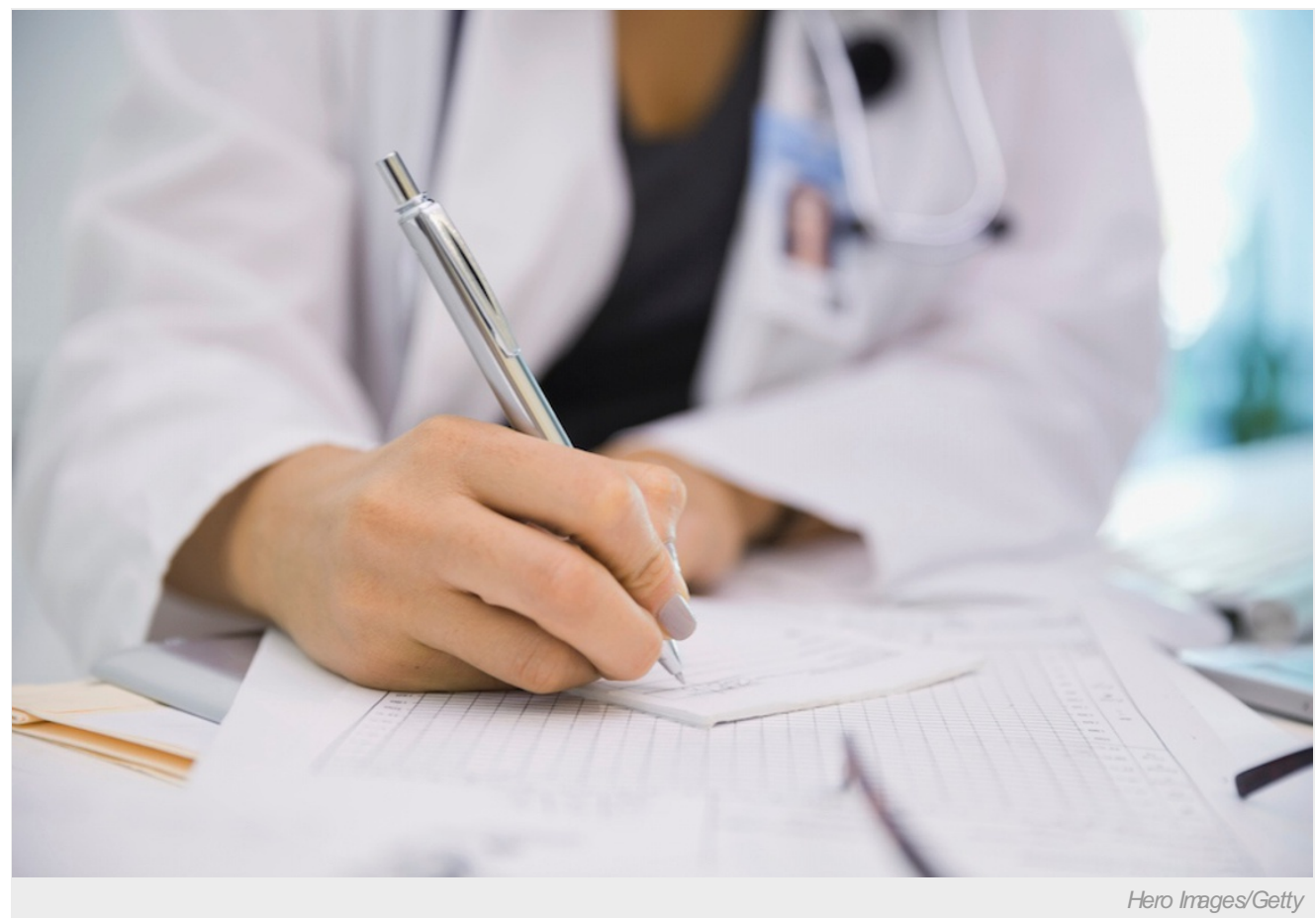

More than $30 \%$ of cancer drugs are prescribed to treat conditions for which they have not been specifically approved.

As the costs of genetic sequencing fall, oncologists are starting to prescribe expensive new drugs that target the genetic profiles of their patients' tumours, even when those treatments have not been approved for the particular cancer involved.

But such 'off-label' use is running ahead of the state of scientific knowledge, suggests the first randomized clinical trial to test the idea. The study, published in Lancet Oncology, found that using personalized cancer drugs off-label provides no benefit over conventional chemotherapy ${ }^{1}$.

"This study is important because many oncologists have already adopted the personalized approach," says Daniel Catenacci, an oncologist at the University of Chicago, Illinois, who was not involved in the trial. "Why have they abandoned the science?"

Lead author of the study Christophe Le Tourneau, an oncologist at the Curie Institute in Paris, says that he sees such off-label treatments "quite often" in practice. "I understand why it happens: patients want to live and physicians want to offer help," he says. But Le Tourneau adds that patients whose tumours have genetic alterations that might be targeted by a non-approved drug are better served by entering clinical trials.

\section{Precision treatments}

A small but growing number of personalized cancer drugs have been approved for treating particular cancers that involve specific mutations, but oncologists hope that these drugs will also work against related mutations in other cancers. By some counts, more than $30 \%$ of cancer drugs are prescribed off-label ${ }^{2,3}$.

To test the benefits of off-label tailored drug regimens, researchers at eight French hospitals analysed their patients' tumours to look for genetic or molecular abnormalities that might be amenable to precision medicine. The researchers randomly assigned 195 suitable patients either to one of 10 potentially relevant targeted treatment regimens, or to chemotherapy. There was no significant difference between the effects of the treatments. 
Apostolia Tsimberidou, an oncologist at the University of Texas MD Anderson Cancer Center in Houston, says the study proves that it is feasible to run randomized trials of personalized medicine. But, in her view, the trial was poorly designed. It enrolled patients with advanced disease who were unlikely to benefit, she points out, and many patients received hormone therapy, which purists would not rate as truly targeted therapy. The trial also didn't choose the best possible drugs to evaluate, and relied on a simplistic approach to matching cancer mutations to targeted treatment, she says.

Le Tourneau concedes the points, but notes that better drugs were not commercially available when the trial was set up in 2011 . And he argues that many oncologists make similarly simple decisions when they prescribe off-label personalized drugs.

Catenacci agrees that many cancer physicians, for now, will be no better than the trial at matching possible targeted drugs to cancer mutations. The case for personalized medicine might become stronger if trials can get better at picking the most appropriate treatment to suit particular genetic and molecular signatures, he says.

\section{Personalized cancer trials}

A few such trials are under way. In 2012, Tsimberidou reported that an observational analysis of patients in clinical trials at her institute showed that enrolment in trials of targeted treatments conferred a benefit over prescribing non-targeted agents ${ }^{4}$. She is now working to validate these results in a 1,400-patient randomized trial.

And in June, the US National Cancer Institute announced plans to start enrolling 1,000 patients in a precision-medicine trial called NClMATCH, which will match patients to more than 20 possible drugs on the basis of the genetic abnormalities in the patients' tumours. The American Society of Clinical Oncology has also launched a registry called TAPUR, to compile data on what happens to patients who receive targeted cancer drugs off-label.

If any study does show that it is beneficial to prescribe personalized cancer drugs that target a patient's tumour, regardless of whether the drug has been approved for the particular tissue type involved, that could pose a severe regulatory challenge, says Catenacci.

Le Tourneau adds that it is probably only a matter of time before the field has to face such challenges. Oncologists will eventually tailor treatments to the genetic and molecular profiles of tumours, rather than on the basis of where the tumour appears in the body, he says. "I'm pretty convinced we are getting there."

Nature | doi:10.1038/nature.2015.18389

\section{References}

1. Tourneau, C. L. et al. Lancet Oncol. http://dx.doi.org/10.1016/S1470-2045(15)00188-6 (2015).

2. Conti, R. M. et al. J. Clin. Oncol. 31, 1134-1139 (2013).

3. Joerger, M. et al. Eur. J. Clin. Pharmacol. 70, 719-725 (2014).

4. Tsimberidou, A.-M. et al. Clin. Cancer Res. 18, 6373-6383 (2012). 